(2) Open Access Full Text Article

REVIEW

\title{
Current use and potential role of bevacizumab in the treatment of gastrointestinal cancers
}

\author{
This article was published in the following Dove Press journal: \\ Biologics:Targets \& Therapy \\ 4 September 2009 \\ Number of times this article has been viewed
}

Jia Li

Muhammad Wasif Saif

Yale Cancer Center, Yale School of Medicine, New Haven CT, USA
Correspondence: Muhammad Wasif Saif Associate Professor, Division of Medical Oncology, 333 Cedar Street, FMP II 6, New Haven, CT 06520, USA Email wasif.saif@yale.edu

\begin{abstract}
Angiogenesis is essential for cancer growth and metastasis. Vascular endothelial growth factor (VEGF) is a key modulator of angiogenesis. In addition, overexpression of VEGF is correlated with advanced disease and poor prognosis. Bevacizumab, a recombinant humanized anti-VEGF monoclonal antibody, is the first anti-angiogenic agent approved by Food and Drug Administration for use in treatment of human solid cancers. Although bevacizumab has received most attention for first-line treatment of advanced colorectal and nonsmall-cell lung cancer, there is a rapidly growing body of evidence for its efficacy in treatment of a number of other solid tumors. We present the current status and potential use of bevacizumab therapy in gastrointestinal cancers.

Keywords: advanced colon cancer, angiogenesis, bevacizumab, chemotherapy, metastatic, targeted therapy, vascular endothelial growth factor, gastric cancer, pancreatic cancer, hepatocellular cancer
\end{abstract}

\section{Development of bevacizumab}

Angiogenesis has been known to be a critical process in tumorigenesis and metastases. ${ }^{1}$ Vascular endothelial growth factor (VEGF), a pro-angiogenic factor, binds to two receptos VEGFR-1 (Flt-1) and VEGFR-2 (Flk-1/KDR), activates receptor tyrosine kinase (RTK) activity and induces angiogenesis. ${ }^{1}$ VEGF and its receptors are often overexpressed in tumors. ${ }^{2-5}$ Therefore, targeting angiogenesis was proposed by Folkman et al as a therapeutic strategy in the 1970s. ${ }^{6-10}$ Approaches to block the VEGF signaling pathway fall into two categories: anti-VEGF mAB (eg, bevacizumab) and RTK inhibitors (RTKi, eg, sunitinib and sorafenib). ${ }^{11-13}$

Bevacizumab (Avastin ${ }^{\circledR}$; Genentech/Roche) is a recombinant humanized $\mathrm{mAB}$ VEGF (VEGF-A for human variant), which theoretically blocks all isoforms of VEGFs to bind to VEGF receptors. ${ }^{14}$ Bevacizumab was approved by the Food and Drug Administration (FDA) in 2004 for use in metastatic colorectal cancer (mCRC) when used with fluorouracil-containing regimens. ${ }^{15}$ Its approval was subsequently extended to nonsquamous nonsmall cell lung cancer and breast cancer in 2006 and $2008 .{ }^{16,17}$

Clinical studies of bevacizumab are underway in early stage colon cancer, unresectable hepatocellular carcinoma (HCC), pancreatic cancer, neuroendocrine tumors, nonmetastatic breast cancer, renal cell carcinoma, glioblastoma multiforme, ovarian cancer, and castrate resistant prostate cancer. ${ }^{18,19}$ This review article will focus on the current use and potential role of bevacizumab in gastrointestinal malignancies. 


\section{Colorectal cancer (CRC)}

Colorectal cancer, the third-leading cause of cancer-related death in the United States, kills approximately 50,000 patients per year. ${ }^{20}$ The mortality rate has slightly decreased in recent decades. In addition to public awareness and early detection, effective adjuvant therapies are significant players in improving the outcome.

\section{Bevacizumab in first-line therapy for advanced CRC (Table I)}

Four cytotoxic chemotherapy regimens are generally accpeted as first line therapies for mCRC: fluouracil and leucovorin (5-FU/LV), 5-FU/LV/oxaliplatin (FOLFOX), 5-FU/LV/irinotecan (FOLFIRI), and capecitabine/oxaliplatin (CapeOX, also named XelOX). ${ }^{21-25}$

The first phase II trial investigating the safety and efficacy of bevacizumab in the first-line treatment of mCRC was conducted in 2003. ${ }^{26}$ One hundred and four patients with untreated $\mathrm{mCRC}$ randomized to receive one of the three regimens: 5-FU/LV (control arm), 5-FU/LV plus lowdose bevacizumab $(5 \mathrm{mg} / \mathrm{kg})$ and 5-FU/LV plus high-dose bevacizumab $(10 \mathrm{mg} / \mathrm{kg})$. The addition of bevacizumab to $5-\mathrm{FU} / \mathrm{LV}$ increased the response rate (RR), prolonged median time to disease progression (TTP), and median overall survival (OS) (Table 1). Surprisingly, the higher dose did not correlate with higher efficacy. Bevacizumab-related toxicities were thrombosis, hypertension, proteinuria, and epistaxis.

Pooled results from several phase II studies have subsequently demonstrated that adding bevacizumab to $5-\mathrm{FU} / \mathrm{LV}$ regimens improved $\mathrm{OS}$ in untreated $\mathrm{mCRC}$ from 14.6 to 17.9 months. ${ }^{26-29}$

The encouraging phase II result led to randomized large phase III trials with the goal of incorporating bevacizumab into first-line therapy for mCRC. 'Low-dose' bevacizumab $(5 \mathrm{mg} / \mathrm{kg}$ ) was used in combination with irinotecan, $5-\mathrm{FU}$, and LV (IFL) in the pivotal phase III trial. ${ }^{30}$ Eight hundred and thirteen patients with untreated $\mathrm{mCRC}$ received either IFL plus placebo, or IFL plus bevacizumab. This trial includes approximately $20 \%$ of all patients with diagnosis of rectal cancer. IFL plus bevacizumab combination was proved to be superior to IFL plus placebo not only in RR (44.8\% vs $34.8 \%, P=0.004)$ but also in OS with an absolute benefit of 4.7 months (20.3 vs 15.6 months, $P<0.001$ ), as well as progression-free survival (PFS) with an absolute benefit of 4.4 months (10.6 vs 6.2 months, $P<0.001$ ). Safety data in this trial revealed manageable hypertension related to bevacizumab, but no thromboembolic events or proteinuria.

This was the first time that a large trial confirmed the role of bevacizumab in prolonging OS in $\mathrm{mCRC}$ in a clinically meaningful way. This trial led to FDA approval of bevacizumab in first-line setting in combination with fluorouracilbased chemotherapy. ${ }^{15}$

The efficacy of bevacizumab in irinotecan-containing regimens was also tested in phase III trial. Fuchs and colleagues initially designed a phase III trial (BICC-C) to compare the efficacy of 3-irinotecan containing regimens in the first-line treatment of mCRC: irinotecan plus infusional (FOLFIRI), bolus (mIFL) or oral fluoropyrimidine (CapeIRI). ${ }^{31} 430$ patients were enrolled into this trial. After FDA approved bevacizumab in 2004, this trial was amended to add 117 patients to receive either FOLFIRI plus bevacizumab $(n=57)$ or mIFL plus bevacizumab $(n=60)$, no further enrollment was made to CapeIRI arm due to toxicity concerns. ${ }^{31,32}$ After a median follow-up of 34.4 months, the data demonstrated superior activity of FOLFIRI plus bevacizumab over mIFL plus bevacizumab in terms of OS (28 vs 19.2 months; $P=0.037$ ), and 1-year survival rate $(87 \%$ vs $61 \%$; respectively). However, both regimens achieved similar overall response rate $(57.9 \%$ for FOLFIRI plus bevacizumab and $53.3 \%$ for mIFL plus bevacizumab). This trial proved the efficacy of bevacizumab in combination with irinotecan-containing regimens, more importantly, this trial established the standard of care of infusional 5-FU for the irinotecan-based regimen.

Another large study, NO16966 which originally designed to compare the noninferiority of CapeOX to FOLFOX-4,

Table I Summary of the phase II trial investigating two doses of bevacizumab plus 5-FU/LV

\begin{tabular}{|c|c|c|c|}
\hline & 5-FU/LV & $\begin{array}{l}5-\mathrm{FU} / \mathrm{LV}+\text { bevacizumab } \\
(5 \mathrm{mg} / \mathrm{kg})\end{array}$ & $\begin{array}{l}\text { 5-FU/LV + bevacizumab } \\
(10 \mathrm{mg} / \mathrm{kg})\end{array}$ \\
\hline Response rate & $17 \%$ & $40 \%(* P=0.029)$ & $24 \%(* P=0.434)$ \\
\hline mTTP & 5.2 months & 9.0 months $(* P=0.005)$ & 7.2 months $(* P=0.217)$ \\
\hline mOS & I3.8 months & 21.5 months $(* P=0.137)$ & 16.1 months $(* P=0.582)$ \\
\hline
\end{tabular}

*Compared with 5-FU/LV control arm.

Abbreviations: $\mathrm{mOS}$, median overall survival; mTTP, median time to progression; 5-FU/LV, fluouracil and leucovorin; FOLFOX, 5-FU/LV/oxaliplatin; mFOLFOX, modified FOLFOX; FOLFIRI, 5-FU/LV/irinotecan; CapeOX, capecitabine/oxaliplatin; IFL, irinotecan, 5-FU, and LV; mIFL, bolus IFL. 
was modified to test the additional benefit of bevacizumab in first-line therapies after the aforementioned phase III trial demonstrated superiority of adding bevacizumab into first-line cytotoxic chemotherapy. Therefore, this trial included 4 arms in order to compare the efficacy of CapeOX and FOLFOX-4 with or without bevacizumab..$^{33} \mathrm{~A}$ total of 1401 patients were enrolled. Bevacizumab arms achieved significantly longer PFS compared with non-bevacizumab arms (9.4 vs 8.0, hazard ratio $[\mathrm{HR}]=0.83 ; 97.5 \%$ confidence interval $[\mathrm{CI}]$, 0.72 to $0.95 ; P=0.0023$ ). However, the addition of bevacizumab did not affect OS (21.3 vs 19.9 months, HR $=0.89$; $97.5 \% \mathrm{CI}, 0.76$ to $1.03 ; P=0.077)$. The authors argued the lack of OS benefit might be caused by early discontinuation of bevacizumab. Nevertheless, this trial is still considered a clinically meaningful study. The National Comprehensive Cancer Network of the United States (NCCN) recommend bevacizumab be added with front-line oxaliplatin-containing regimens for initial treatment.

Besides FOLFOX-4, bevacizumab was also investigated in combination with other three oxaliplatin-containing regimens in Three Regimens of Eloxatin Evaluation (TREE)-2 study. ${ }^{34}$ In this study, oxaliplatin with infusional, bolus, and oral fluoropyrimidine with or without bevacizumab were evaluated as first-line treatment for $\mathrm{mCRC}$. The addition of bevacizumab to all three regimens improved overall RR and prolonged OS compared with their comparators. Among these three regimens, oxaliplatin plus infusional 5-FU/LV (FOLFOX) plus bevacizumab appeared to be superior to the other two (Table 2).

Bevacizumab in combination with another oxaliplatincontaining regimen, modified (m) FOLFOX-7, was investigated CONcePT (Combined Oxaliplatin Neurotoxicity Prevention Trial) Trial. ${ }^{35}$ This trial was designed to optimize oxaliplatin dose in order to reduce neurotoxicity. Therefore, the major two arms are alternating mFOLFOX-7 with 5-FU/LV plus bevacizumab, and continuous mFOLFOX-7 plus bevacizumab. The primary endpoint was time to treatment failure. CONcePT also measured the effect of intravenous calcium and magnesium supplement on neurotoxicity prevention. After 140 patients with $\mathrm{mCRC}$ were enrolled, the first interim analysis suggested calcium/magnesium supplement might be negatively impacting anti-tumor activity of chemotherapy. Therefore, this trial was closed early in June 2007. However, an independent radiology review demonstrated that response was not affected by calcium/magnesium supplementation. The intermittent oxaliplatin arm showed significant improvement of PFS and time to treatment failure (TTF) when compared with the continuous oxaliplatin arm (12 vs 7.3 months, $P=0.03$; and 5.6 vs 4.2 months, $P=0.003$; respectively). This trial proved the convincing benefit of intermittent oxaliplatin over continuous oxaliplatin. However, the role of bevacizumab was impossible to be investigated given both arms contain same dose/schedule of bevacizumab.

\section{Bevacizumab in second-line therapy for advanced CRC}

Bevacizumab was also tested in $\mathrm{mCRC}$ in combination with oxaliplatin-based regimen in second-line setting. In the Eastern Cooperative Oncology Group (ECOG) trial (E3200), 829 patients with previously treated CRC but without prior oxaliplatin or bevacizumab exposure were

Table 2 Summary of trials investigating bevacizumab in first-line setting for metastatic colorectal cancer

\begin{tabular}{|c|c|c|c|c|c|}
\hline Trial/Reference & No. of patients & Regimens & ORR & mPFS (months) & mOS (months) \\
\hline Hurwitz trial ${ }^{30}$ & 813 & IFL + bevacizumab vs IFL + placebo & $\begin{array}{l}44.8 \% \text { vs } 34.8 \% \\
(P=0.004)\end{array}$ & 10.6 vs $6.2(P<0.00 \mathrm{I})$ & 20.3 vs $15.6(P<0.001)$ \\
\hline $\mathrm{BICC}-\mathrm{C}^{31,32}$ & 117 & $\begin{array}{l}\text { FOLFIRI + bevacizumab }(n=57) \\
\text { vs mIFL + bevacizumab }(n=60)\end{array}$ & $57.9 \%$ vs $53.3 \%$ & $\begin{array}{l}\text { I I. } 2 \text { vs } 8.3 \\
\text { (not statistically } \\
\text { significant) }\end{array}$ & 28 vs $19.2 \%(P=0.037)$ \\
\hline $\mathrm{NO} 16966^{33}$ & 1401 & $\begin{array}{l}\text { CapeOX/FOLFOX } 4+\text { placebo } \\
(n=70 I) \text { vs CapeOX/FOLFOX-4 + } \\
\text { bevacizumab }(n=699)\end{array}$ & $49 \%$ vs $47 \%$ & 8.0 vs $9.4(P=0.023)$ & 19.9 vs $21.3(P=0.077)$ \\
\hline TREE- $2^{34}$ & 213 & $\begin{array}{l}\text { mFOLFOX + bevacizumab }(n=7 \mathrm{I}) \\
\text { vs bFOL + bevacizumab }(n=70) \\
\text { vs CapeOx + bevacizumab }(n=72)\end{array}$ & $52 \%$ vs $39 \%$ vs $46 \%$ & 9.9 vs 8.3 vs 10.3 & 26.1 vs 20.4 vs 24.6 \\
\hline $\mathrm{CONcePT}^{35}$ & 140 & $\begin{array}{l}\text { mFOLFOX-7 + bevacizumab vs } \\
\text { mFOLFOX-7/5-FU/LV + bevacizumab }\end{array}$ & Not available & 12 vs $7.3(P=0.03)$ & Not available \\
\hline
\end{tabular}

Abbreviations: ORR, overall response rate; mPFS, median progression-free survival; mOS, median overall survival; 5-FU/LV, fluouracil and leucovorin; FOLFOX, 5-FU/LV/ oxaliplatin; mFOLFOX, modified FOLFOX; FOLFIRI, 5-FU/LV/irinotecan; CapeOX, capecitabine/oxaliplatin; IFL, irinotecan, 5-FU, and LV; mIFL, bolus IFL. 
randomized to 3 arms at 1:1:1 ratio: FOLFOX-4 plus bevacizumab, FOLFOX-4, or bevacizumab alone. ${ }^{36}$ The dose of bevacizumab chosen was $10 \mathrm{mg} / \mathrm{kg}$ although a phase II trial concluded that $5 \mathrm{mg} / \mathrm{kg}$ dose was more effective than $10 \mathrm{mg} / \mathrm{kg}$ dose. ${ }^{26}$ The authors of E3200 trial believed that the effect of bevacizumab was dose-dependent and $10 \mathrm{mg} / \mathrm{kg}$ should be more active in combination with chemotherapy. FOLFOX-4 plus bevacizumab treatment in irinotecan-refractory mCRC demonstrated not only an improved overall RR but also statistically significant survival benefit compared with FOLFOX-4 or bevacizumab alone (Table 3).

Interestingly, prior to the publication of this trial, the study of FOLFOX-4 as first-line therapy for mCRC was just accepted as the standard of care for initial treatment in the United States. ${ }^{25}$ Therefore, the E3200 trial also proved the anti-tumor effect of FOLFOX-4 with or without bevacizumab in second-line setting after irinotecan failure. More importantly, bevacizumab in combining with cytotoxic chemotherapy provided additional response and survival benefit.

Similarly, bevacizumab was investigated in combination with an irinotecan-based regimen in second-line setting. Bowel Oncology and Cetuximab Antibody (BOND)-1 study conducted by Cunningham et al had established the role of irinotecan and epithelial growth factor receptor (EGFR) monoclonal antibody (mAb)-cetuximab in secondline setting. ${ }^{37}$ The BOND-2 study was subsequently designed to evaluate any benefit of adding bevacizumab to irinotecan-cetuximab combination therapy. ${ }^{38}$ Until now, this was the first and remained the only trial showing that concurrent use of dual-mAb therapy did not increase toxicity but improved PFS. Unfortunately, these results were not shown in the large trials Panitumumab Advanced Colorectal Cancer Evaluation (PACCE) or CApecitabine, IRinotecan, and Oxaliplatin in advanced CRC (CAIRO)-2 studies, ${ }^{39-52}$ both of which showed unacceptable toxicities when 2 monoclonal antibodies were administered in combination with cytotoxic

Table 3 Summary of the efficacy data of E3200 trial ${ }^{36}$

\begin{tabular}{lllll}
\hline & $\begin{array}{l}\text { FOLFOX-4 } \\
\text { bevacizumab } \\
(\mathbf{n}=\mathbf{2 8 6})\end{array}$ & $\begin{array}{l}\text { FOLFOX-4 } \\
(\mathbf{n}=\mathbf{2 9 1})\end{array}$ & $\begin{array}{l}\text { Bevacizumab } \\
(\mathbf{n}=\mathbf{2 4 3})\end{array}$ & $P$ value \\
\hline ORR & $22.7 \%$ & $8.6 \%$ & $3.3 \%$ & $P<0.001$ \\
PFS & 7.3 months & 4.7 months & 2.7 months & $P<0.00 \mathrm{I}$ \\
OS & 12.7 months & 10.8 months & 10.2 months & $P=0.001 \mathrm{I}$ \\
\hline
\end{tabular}

Abbreviations: ORR, overall response rate; $\mathrm{PFS}$, progression-free survival; OS, overall survival; 5-FU/LV, fluouracil and leucovorin; FOLFOX, 5-FU/LV/oxaliplatin; mFOLFOX, modified FOLFOX; FOLFIRI, 5-FU/LV/irinotecan; CapeOX, capecitabine/oxaliplatin; IFL, irinotecan, 5-FU, and LV; mIFL, bolus IFL. chemotherapy. Dual biologic therapy will be discussed in another section in this review.

There are well-accepted convincing data of incorporating bevacizumab with irinotecan-, oxaliplatin-based regimens as first-line or second-line therapies for mCRC. However, many clinical questions remained unanswered, such as the role of bevacizumab maintenance, or continuation of bevacizumab in second- or third-line settings in patients who had prior exposure to bevacizumab-containing regimens. The DREAM (Double Reintroduction with Erlotinib and Avastin in Metastatic CRC) trial was designed to evaluate the role of bevacizumab with or without erlotinib as maintenance treatment. ${ }^{43}$ Patients were treated with 6 cycles of FOLFOX-7 plus bevacizumab or 6 cycles of CapeOX-4 plus bevacizumab, then maintenance bevacizumab with or without erlotinib, followed by reinduction of FOLFOX-7 or CapeOX plus bevacizumab on disease progression. The primary endpoints are PFS and OS. We hope the final data will be able to answer the above questions.

Current consensus does not support the continuation of bevacizumab if a patient has already had it in first-line therapy, because of lack of supporting data.

\section{Dual-biologic therapy in $\mathrm{mCRC}$}

In theory, targeting two separate pathways such as VEGF and EGF pathways simultaneously potentially produces higher anti-tumor effect. However, in reality, we have seen extremely dangerous toxicities in several trials using $2 \mathrm{mAbs}$ or $1 \mathrm{mAb}$ plus $1 \mathrm{RTKi} .{ }^{39-42}$

\section{Combination of bevacizumab and EGFR mAb}

The first trial combining bevacizumab with another biologic agent was BOND-2 study. ${ }^{38} \mathrm{~A}$ regimen of irinotecan, cetuximab plus bevacizumab was investigated. The efficacy data demonstrated that the dual-biologic regimen potentially provided survival benefit compared with mono-biologic regimen (such as irinotecan/cetuximab) and could be considered as a second-line option for irinotecan-refractory mCRC.

However when a similar idea was tested in large trials, an unexpectedly high incidence of severe toxicity emerged. The PACCE trial was designed to investigate the role of combining anti-EGFR and anti-VEGF antibody with chemotherapy as first-line treatment in patients with advanced CRC. ${ }^{39,40}$ In this study, FOLFOX or FOLFIRI plus bevacizumab with or without panitumumab, an anti-EGFR mAb, were compared. The rationale for this design was based on preclinical studies suggestive of additive anti-tumor effect of targeting on 2 separate pathways. The data from the PACCE trial were 
certainly surprising. The dual-biologic target therapy did not provide any benefit to the efficacy but significantly increased the incidence of severe gastrointestinal (GI) toxicity such as diarrhea and infections.

CAIRO-2 study conducted by the Dutch Colorectal Cancer Group is another large phase III trial attempting to evaluate the effectiveness of dual biologic therapy. ${ }^{41}$ This is a well-designed, multicenter clinical trial in which 736 patients with previously untreated mCRC were randomly assigned to receive capecitabine, oxaliplatin, and bevacizumab (CB) with or without cetuximab (CBC) every 3 weeks. Besides the use of bevacizumab, there are 2 differences between CAIRO-2 and PACCE: panitumumab was changed to a more commonly used anti-EGFR antibody, cetuximab; the backbone conventional chemotherapy switched from FOLFOX/ FOLFIRI to CapeOX. The final results of CAIRO-2 were recently published (Table 4$).{ }^{42}$ The results were somewhat expected - reduced survival length plus overwhelming toxicities.

The two large trials, PACCE and CAIRO-2, both showed significant toxicity without any clinical benefit when combining 2 monoclonal antibodies with cytotoxic chemotherapy. Until now, there is no clear explanation for the observed negative effect. Therefore, the National Cancer Institute (NCI) suspended another ongoing large trial being conducted by the Cancer and Leukemia Group B (CALGB) and South West Oncology Group (SWOG). ${ }^{44}$ The study was designed to answer the question of whether 2 antibodies are superior to single antibody when combined with chemotherapy. The current consensus is that combined biologic therapy with anti-EGFR and anti-VEGF antibodies should not be used outside an appropriate clinical trial setting. We have known that neither cetuximab nor panitumumab would provide any

Table 4 Toxicity and efficacy profile of CAIRO-2 study ${ }^{41}$

\begin{tabular}{llll}
\hline & $\begin{array}{l}\text { CB } \\
(\mathbf{n}=\mathbf{3 6 8})\end{array}$ & $\begin{array}{l}\text { CBC } \\
(\mathbf{n}=\mathbf{3 6 8})\end{array}$ & P value \\
\hline Any grade 3 or above toxicity (\%) & 73.2 & 81.7 & 0.006 \\
Any cutaneous event (\%) & 20.8 & 39.1 & $<0.001$ \\
Diarrhea (\%) & 19.1 & $26 \%$ & 0.03 \\
Response rate (\%) & 50 & 52.7 & 0.49 \\
mPFS (months) & 10.7 & 9.4 & 0.16 \\
mOS (months) & 20.3 & 19.4 & 0.49 \\
Disease control rate (\%) & 94 & 94.6 & 0.72 \\
\hline
\end{tabular}

Abbreviations: $C B$, capecitabine, oxaliplatin and bevacizumab; $C B C$, capecitabine, oxaliplatin and bevacizumab with or without cetuximab; mPFS, median progression-free survival; mOS, median overall survival; 5-FU/LV, fluouracil and leucovorin; FOLFOX, 5-FU/LV/oxaliplatin; mFOLFOX, modified FOLFOX; FOLFIRI, 5-FU/LV/irinotecan; CapeOX, capecitabine/oxaliplatin; IFL, irinotecan, 5-FU, and LV; mIFL, bolus IFL. response benefit in KRAS mutant CRC. Nevertheless, the negative outcome could not be all attributed to the $K R A S$ status.

\section{Combination of bevacizumab and erlotinib}

Bevacizumab and erlotinib were combined with FOLFOX for first-line treatment of $\mathrm{mCRC}$ in a phase II study. ${ }^{45} \mathrm{All}$ 35 patients initially enrolled for intention to treat were off study secondary to intolerable toxicity rather than disease progression. Major toxicities included rash, diarrhea, and neuropathy. Interestingly, despite premature termination of this trial because of toxicity, 12 patients achieved objective response, and 1 patient even had complete response. However, interpreting these efficacy data in an early closed trial would be challenging. Overall this double-target therapy combination was believed to be too toxic.

\section{Bevacizumab in CRC adjuvant setting}

Should we extrapolate the promising data of bevacizumab in advanced $\mathrm{CRC}$ into adjuvant setting? The answer to this question is no for now. Clearly current data from the National Surgical Adjuvant Breast and Bowel Project C-08 trial (NSABP C-08) did not support the use of bevacizumab in the adjuvant setting given lack of survival benefit. Allegra and colleagues published the safety data. ${ }^{46}$ This randomized phase III trial was designed to compare modified FOLFOX-6 (every 2 weeks for 12 cycles) with bevacizumab vs without bevacizumab. For the nonbevacizumab arm, patients received standard mFOLFOX-6 for a total of 12 cycles, while patients in the bevacizumab arm were offered bevacizumab maintenance after completion of 12 cycles of mFOLFOX-6 plus bevacizumab (Figure 1).

The safety data were obtained from 2647 patients with surgically resected stage II/III colon cancer. The dose of bevacizumab was $5 \mathrm{mg} / \mathrm{kg}$ every 2 weeks. The primary endpoint is 3-year disease-free survival (DFS). Demographic factors were well balanced. Twenty-five percent of patients had stage II disease in each arm.

The safety data revealed virtually identical incidence of grade 4 toxicities in both arms $(15.2 \%$ and $15.0 \%$, respectively). Bevacizumab arm did show significantly higher rate of several toxicities; however, those previously concerned toxicities such as GI bleeding, perforation, or ischemic events were not statistically different when compared with the nonbevacizumab arm (Table 5).

Following the overall acceptable safety data, the final survival data were subsequently presented at the 2009 ASCO annual meeting. ${ }^{47}$ Surprisingly, no PFS benefit was observed 


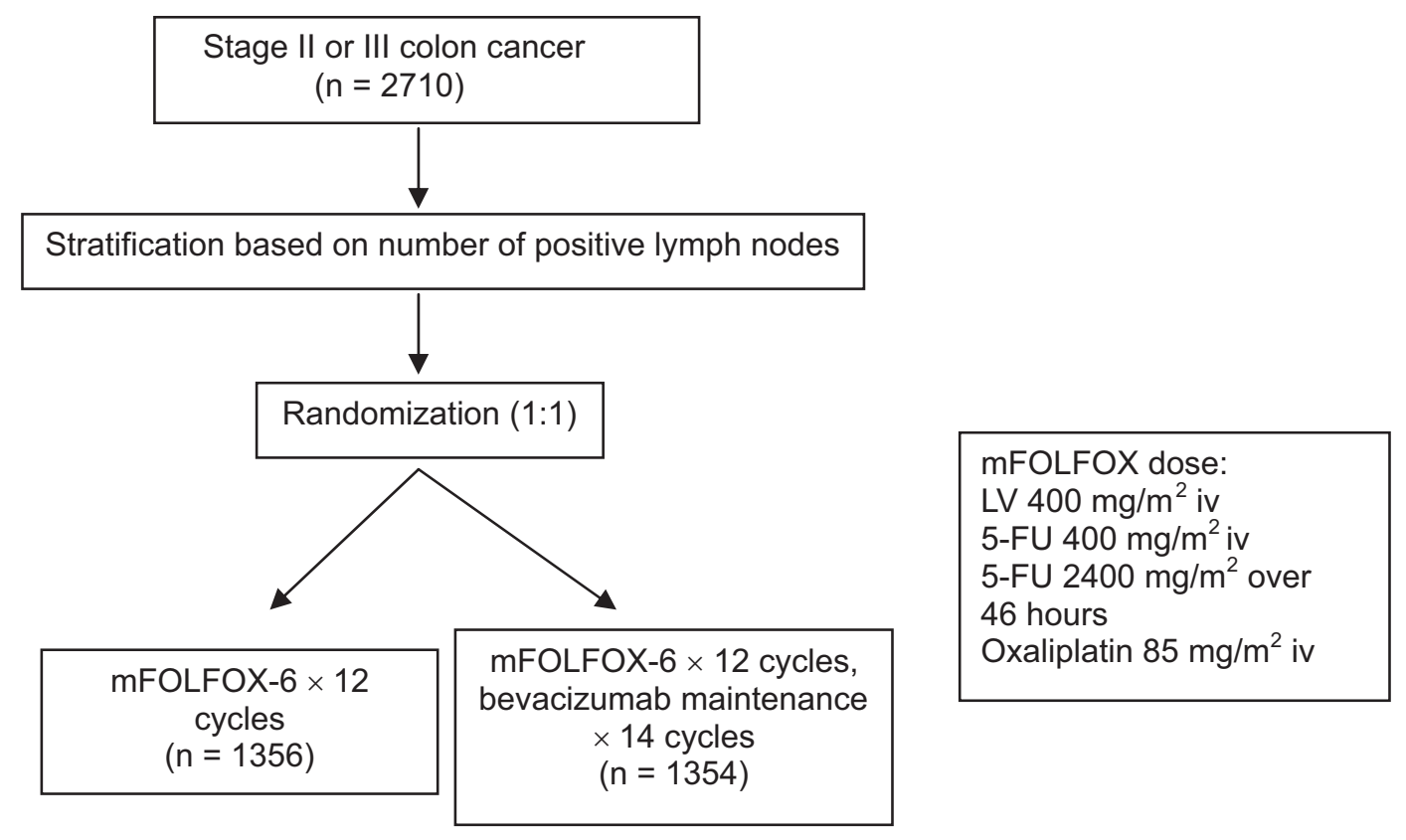

Figure I Schema of NSABP C-08 trial..$^{46,47}$

with the addition of bevacizumab regardless of disease stage (Table 6). Therefore, use of bevacizumab in the colon adjuvant setting cannot currently be recommended in the absence of efficacy data.

\section{Pancreatic cancer}

Pancreatic cancer is the fourth leading cause of cancer related death in the US. ${ }^{48}$ The prognosis of pancreatic cancer depends on the resectability of primary tumor; however, more than $80 \%$ of patients have locally advanced disease or metastases upon diagnosis. Single agent gemcitabine remains the only universally accepted effective palliative treatment that not only provides clinical benefit but also prolongs OS. ${ }^{49}$

Since the approval of gemcitabine in 1997 by the FDA, numerous combinations using gemcitabine as a backbone were investigated in many clinical trials. The combination of erlotinib with gemcitabine reached statistical significance in terms of OS, leading to FDA approval of erlotinib in advanced pancreatic cancer (APC) in November 2005. ${ }^{50}$

\section{Use of bevacizumab in phase II clinical trials}

Bevacizumab was tested in several combinations with cytotoxic chemotherapy in phase II trials. Kindler et al published the first phase II trial of bevacizumab in combination with gemcitabine in untreated APC as first-line therapy. ${ }^{51}$ Gemcitabine $\left(1000 \mathrm{mg} / \mathrm{m}^{2}\right)$ was given on days 1,8 , and 15 every 28 days; bevacizumab $(10 \mathrm{mg} / \mathrm{kg})$ was given on days 1 and 15 after gemcitabine. Fifty-two patients with confirmed APC were treated. Eleven patients $(21 \%)$ had partial responses, and median OS and PFS were 8.8 and 5.4 months, respectively. These results appear encouraging, but the toxicity profile was worrisome. In this 52-patient trial, the incidence of bowel perforation was $8 \%$, which was strikingly high compared to the observations in other colorectal trials.

The North Central Cancer Treatment Group (NCCTG) investigated bevacizumab in combination with gemcitabine

Table 5 Summary of severe toxicity data of NSABP C-08 trial ${ }^{45}$

\begin{tabular}{llll}
\hline $\begin{array}{l}\text { Grade } 3 \text { or above } \\
\text { adverse events }\end{array}$ & $\begin{array}{l}\text { mFOLFOX }+ \\
\text { bevacizumab } \\
(\mathbf{n}=\mathbf{1 3 2 6})\end{array}$ & $\begin{array}{l}\text { mFOLFOX } \\
\text { alone } \\
(\mathbf{n}=\mathbf{1 3 2 1})\end{array}$ & P value \\
\hline Hypertension & $12 \%$ & $1.8 \%$ & $<0.0001$ \\
Wound complications & $1.7 \%$ & $0.3 \%$ & $<0.001$ \\
Pain & $11.1 \%$ & $6.3 \%$ & $<0.000$ I \\
Proteinuria & $2.7 \%$ & $0.8 \%$ & $<0.001$ \\
Neuropathy & $16.7 \%$ & $14.4 \%$ & $\mathrm{NS}$ \\
Gl perforation & $0.3 \%$ & $0.2 \%$ & $\mathrm{NS}$ \\
Any hemorrhage & $1.9 \%$ & $1.9 \%$ & $\mathrm{NS}$ \\
Peripheral arterial & $0.0 \%$ & $0.2 \%$ & $\mathrm{NS}$ \\
ischemia & & & \\
\hline
\end{tabular}

Abbreviations: GI, gastrointestinal; NS, not statistically significant; 5-FU/LV, fluouracil and leucovorin; FOLFOX, 5-FU/LV/oxaliplatin; mFOLFOX, modified FOLFOX; FOLFIRI, 5-FU/LV/irinotecan; CapeOX, capecitabine/oxaliplatin; IFL, irinotecan, 5-FU, and LV; mIFL, bolus IFL. 
Table 6 Survival data of NSABP C- $08^{47}$

\begin{tabular}{lllll}
\hline & $\begin{array}{l}\text { mFOLFOX-6 } \\
\text { bevacizumab } \\
(\mathbf{n}=1334)\end{array}$ & $\begin{array}{l}\text { mFOLFOX-6 } \\
\text { alone } \\
(\mathbf{n}=1338)\end{array}$ & $\begin{array}{l}\text { Hazard } \\
\text { ratio } \\
\mathbf{( H R )}\end{array}$ & P value \\
\hline DFS (3-yr) & $77 \%$ & $75.5 \%$ & 0.89 & 0.15 \\
Stage II & $87 \%$ & $85 \%$ & 0.82 & 0.35 \\
Stage III & $74 \%$ & $72 \%$ & 0.90 & 0.25 \\
\hline
\end{tabular}

Abbreviations: DFS, disease-free survival; 5-FU/LV, fluouracil and leucovorin; FOLFOX 5-FU/LV/oxaliplatin; mFOLFOX, modified FOLFOX; FOLFIRI, 5-FU/LV/irinotecan CapeOX, capecitabine/oxaliplatin; IFL, irinotecan, 5-FU, and LV; mIFL, bolus IFL.

and oxaliplatin, ${ }^{52,53}$ a combination that regained attention after several pooled- and meta-analyses demonstrated a small benefit for selected patients with good performance status. ${ }^{54-57}$ Objective RR was $11.3 \%$ in a total of 80 eligible patients. The 6-month survival was $65.0 \%$; median OS and PFS were 8.1 and 5.7 months, respectively. ${ }^{52,53}$

Bevacizumab with or without docetaxel was tested by Astsaturov and colleagues, ${ }^{58}$ although the rationale of this trial was not convincing given the minimal activity of taxanes in pancreatic cancer in general. Bevacizumab was given at $10 \mathrm{mg} / \mathrm{kg}$ every 2 weeks alone or with docetaxel at $35 \mathrm{mg} / \mathrm{m}^{2}$ weekly for 3 weeks followed by 1 week off. Fifteen out of 30 patients experienced severe adverse events including 5 deep venous thromboses and 2 bowel perforations. There was no difference in terms of PFS or OS in either arm. The results of this trial only confirmed an already-known fact that taxanes generally do not have an anti-tumor effect in APC. In addition, single-agent bevacizumab was also proved to lack efficacy in this aggressive disease.

Overall, all the above-mentioned phase II trials except the combination of bevacizumab and gemcitabine failed to demonstrate any convincing benefit compared with historical gemcitabine monotherapy. ${ }^{51-53,58,59}$

\section{Use of bevacizumab in phase III clinical trials}

The high response rate in phase II results for bevacizumab plus gemcitabine provided the rationale for a phase III CALGB 80303 study, in which gemcitabine with or without bevacizumab were compared. ${ }^{60}$ Six hundred and two patients with APC were enrolled into this large placebo-controlled trial, randomized for patients to receive gemcitabine plus bevacizumab (GB) versus gemcitabine plus placebo (GP). The primary endpoint was OS, secondary endpoints were RR, duration of response, PFS, and toxicity. Median OS and PFS of GB/GP were 5.8/6.1 and 4.9/4.7 months, respectively. RR and 1-year survival rate failed to reach statistical difference.
The comparison of phase II and phase III data revealed that difference in performance status, adjuvant treatment, and/or previous thrombotic events might be able to explain the negative outcome. Toxicity profile was not substantially different from any other large bevacizumab trials, including hypertension ( $8 \%$ ), GI perforation $(0.4 \%)$, GI bleeding $(3 \%)$, proteinuria (4\%), and venous thrombosis (9\%). Nevertheless, the addition of bevacizumab to gemcitabine did not offer any meaningful clinical benefit.

Another phase III trial of bevacizumab was the Tarceva \pm Avastin in APC (AViTA) trial, which was unfortunately another negative trial. ${ }^{61}$ AViTA aimed to explore the superiority of gemcitabine/erlotinib plus bevacizumab over gemcitabine/erlotinib plus placebo in APC. The biologic rationale for such a design came from retrospective studies demonstrating an adverse correlation between overexpression of EGFR and VEGF in pancreatic cancer with survival. ${ }^{62,63}$

OS was the primary objective, PFS, disease control rate, and toxicity were secondary objectives. Six hundred and seven patients with metastatic pancreatic cancer were randomized to receive gemcitabine/erlotinib with or without bevacizumab as initial therapy (Figure 2).

Although DFS (from 3.6 to 4.6 months) met statistical significance (HR 0.73, $P=0.0002$ ), no significant prolongation but a trend toward OS benefit was seen in the bevacizumab $\operatorname{arm}(7.1$ vs 6.0 months, HR $0.89, P=0.2087)$. The authors believe this 1-month disease-free survival benefit suggests that a subset of metastatic patients might derive more benefit from anti-angiogenic therapy than others. However, how to identify these subgroup patients remains unclear.

\section{Hepatocellular carcinoma (HCC)}

$\mathrm{HCC}$ is a highly vascularized tumor with poor prognosis. Its incidence is rising in Europe and US as a result of the high prevalence of hepatitis C. ${ }^{48}$ Surgical resection and liver transplantation are the effective curative interventions only for local resectable disease; however, the majority of cases present with advanced stage or metastatic stage. Sorafenib is the only standard of care for advanced HCC, modest benefit at the sacrifice of toxicity made the development of novel agents urgent. ${ }^{64}$ VEGF is found to be aberrantly expressed in HCC and plays a critical role in tumorigenesis as well as disease progression. ${ }^{5}$ The use of anti-angiogenic agents appears to be a promising approach in view of the highly vascular nature of this tumor. Several early phase trials have investigated the role of bevacizumab in the treatment of HCC.

Finn et al established a mouse model to assess the antiangiogenic effect of bevacizumab. Hep 3B cells, a human 


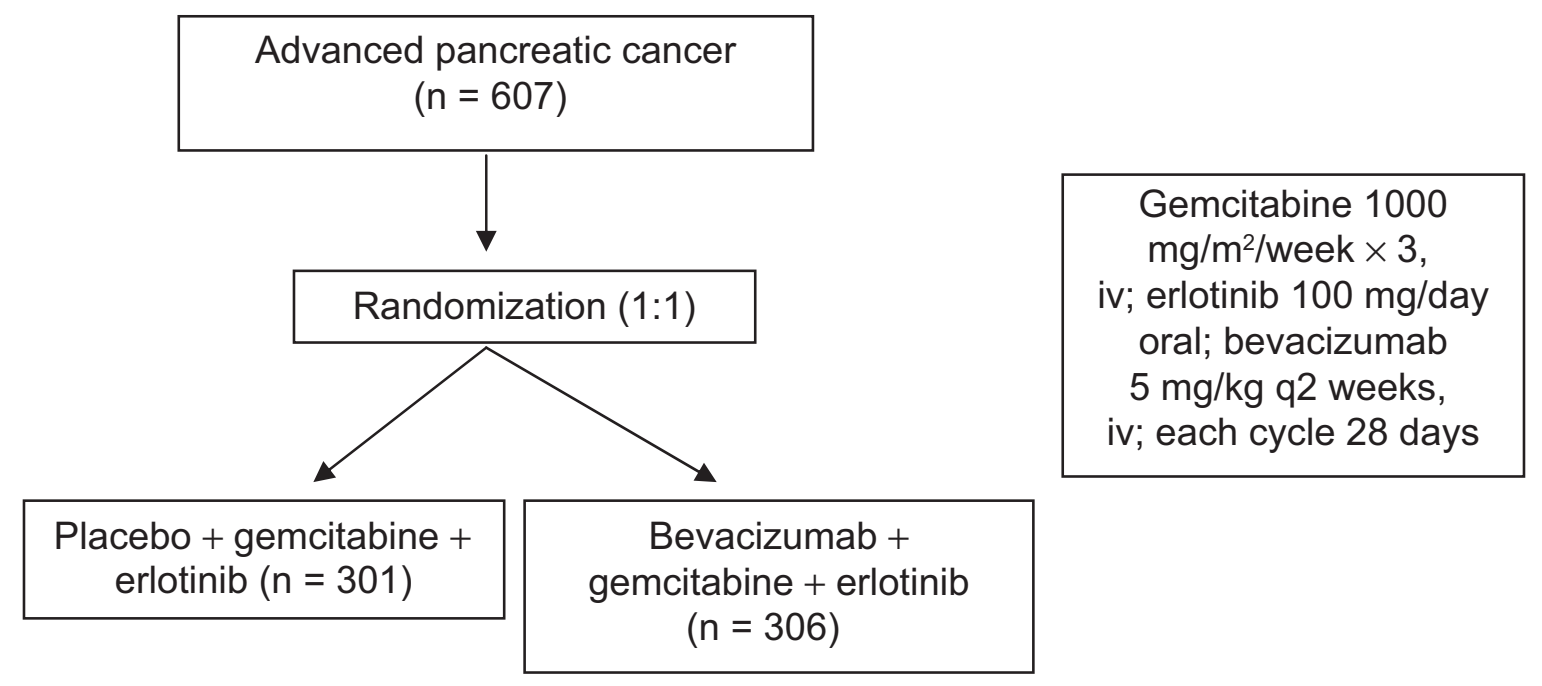

Figure 2 Schema of AViTA trial. ${ }^{61}$

HCC cell line, were inoculated into the livers of severe combined immunodeficient (SCID) mice under anesthesia ${ }^{65}$ Bevacizumab at $5 \mathrm{mg} / \mathrm{kg}$ was administered intraperitoneally twice weekly into half of the mice. Serum alpha-fetoprotein (AFP) and VEGF level were measured as surrogate markers for response, while tumors were harvested for vascular study such as immunohistochemical staining of the endothelial marker CD31. Bevacizumab significantly decreased microvessel density in tumors and decreased serum AFP level. These preclinical data led to bevacizumab being tested in human subjects.

Forty-six patients with unresectable nonmetastatic HCC were enrolled into a phase II study ${ }^{66} 12$ patients received bevacizumab at $5 \mathrm{mg} / \mathrm{kg}$, while the other patients received a higher dose at $10 \mathrm{mg} / \mathrm{kg}$ every 2 weeks until disease progression or treatment-limiting toxicity. Overall objective RR was 13\%. Median PFS was 6.9 months (95\% CI 6.5 to 9.1 months). More than $50 \%$ of patients survived more than 1 year, more than $25 \%$ survived beyond 2 years. The efficacy results were encouraging, but hypertension, bleeding, and thrombotic events remain the major safety concern for bevacizumab treatment.

Zhu et al conducted a phase II trial to examine the efficacy of gemcitabine and oxaliplatin plus bevacizumab (GEMOX-B) in unresectable or metastatic HCC. ${ }^{67}$ The schedule was bevacizumab $10 \mathrm{mg} / \mathrm{kg}$ administered every 14 days, fixed dose rate gemcitabine $1000 \mathrm{mg} / \mathrm{m}^{2}$ every 14 days, and oxaliplatin $85 \mathrm{mg} / \mathrm{m}^{2}$ on the following day after gemcitabine. Thirty patients were eligible for efficacy analysis. Six patients achieved objective response on imaging studies according to RECIST; median PFS and OS were
5.3 and 9.6 months, respectively. Severe toxicities related to treatment were bone marrow suppression, abnormal liver function, hypertension, and fatigue. The benefit derived from this regimen appears to be comparable with that of sorafenib, but a larger trial is warranted for further investigation.

The effect of bevacizumab on angiogenesis was examined by the same research group utilizing computed tomography perfusion scan. ${ }^{68}$ Bevacizumab induced a significant decrease in tumor blood flow, blood volume, and permeability in HCC.

Bevacizumab and erlotinib combination was also investigated in advanced or metastatic HCC at phase II level. ${ }^{69}$ This regimen consists of bevacizumab $10 \mathrm{mg} / \mathrm{kg}$ every 14 days and erlotinib $150 \mathrm{mg}$ oral daily, for each 28-day cycle. The primary endpoint of this phase II study was PFS at 16 weeks. Of 40 patients, $62.5 \%$ survived beyond 16 weeks without evidence of progression. Ten patients achieved a partial response, while median PFS and OS were 9.1 and 15.9 months, respectively. The dual-biologic agent strategy showed very promising anti-tumor activity in HCC. More importantly, toxicity profile of this combination regimen was acceptable. More recently, an updated report was presented at the 2009 ASCO annual meeting. ${ }^{70}$ With a total of 57 patients, overall RR was $28 \%$, and median PFS and OS were 7.9 months and 12.8 months, respectively. PFS at 16 weeks was $73 \%$. Currently, a multi-institution randomized phase II trial is ongoing to further characterize the clinical benefit of the combination.

Among other regimens, the combinations of bevacizumab and mammalian target of rapamycin (mTOR) inhibitors appear encouraging. Bevacizumab and rapamycin combination 
offered promising anti-tumor activity in an orthotopic intrahepatic xenograft mouse model. ${ }^{71}$ Bevacizumab and everolimus combination is also being tested in human subjects. $^{72}$

\section{Cholangiocarcinoma and gallbladder cancer}

Unlike other GI malignancies, there is very limited experience with the use of bevacizumab in cholangiocarcinoma or gallbladder cancer. A Taiwan oncology group reported an interesting case in 2006. An elderly patient with cholangiocarcinoma achieved quick response in liver metastases after being treated with cisplatin, 5-FU, leucovorin plus bevacizumab. ${ }^{73}$ However, this is just a single case observation outside a clinical trial setting. In addition, the effect of tumor shrinking can hardly be attributed to bevacizumab anti-angiogenic effect alone. More prospective studies should address whether this combination can be an appropriate option for advanced biliary duct cancer.

Given the popularity of the double pathway blocking approach, especially the combination of bevacizumab and erlotinib, Holen and colleagues also investigated this combination in previously untreated advanced gallbladder cancer or cholangiocarcinoma. The data were presented at the 2008 ASCO annual meeting. ${ }^{74}$ Twenty-nine patients were eligible for enrollment; 3 out of 17 (17.6\%) evaluable patients achieved partial response (PR). The projected accrual is not completed yet for this trial, and final efficacy data are therefore pending. The data are currently being validated in other larger trials (Table 7). Several other trials are ongoing to investigate the efficacy of bevacizumab with cytotoxic agents, and/or radiation in biliary duct and gallbladder cancer.

\section{Gastric and esophageal cancer}

Gastric cancer is one of the leading causes of cancer-related death in the US. Median OS for metastatic or unresectable disease is usually less than 10 months. Despite response rates of $30 \%$ to $60 \%$ to combination chemotherapy, response duration is usually 4 to 6 months and 24-month survival is $5 \%$ to $10 \%$. VEGF overexpression was also found in gastric cancer and was associated with poor prognosis. ${ }^{75,76}$ This suggests that anti-VEGF therapy might have also have an effect on gastric cancer. In a mouse model with gastric peritoneal metastases, retarded tumor growth and development of malignant ascites were demonstrated after being treated with bevacizumab. ${ }^{77}$

Shah and colleagues conducted a phase II trial to evaluate the efficacy and safety of adding bevacizumab to irinotecan/ cisplatin combination in metastatic or unresectable gastric and gastroesophageal (GE) junction adenocarcinoma. ${ }^{78}$ This is a multicenter trial that enrolled 47 patients. The dose and schedule were: bevacizumab $15 \mathrm{mg} / \mathrm{kg}$ on day 1 , irinotecan $65 \mathrm{mg} / \mathrm{m}^{2}$, and cisplatin $30 \mathrm{mg} / \mathrm{m}^{2}$ on days 1 and 8 every 21 days. With a median follow-up of 12.2 months, this combination regimen achieved overall RR of $65 \%$ (95\% CI $46 \%$ to $80 \%$ ), median TTP of 8.3 months (95\% CI 5.5 to 9.9), and median OS of 12.3 months (95\% CI 11.3 to 17.2), suggesting a $75 \%$ improvement compared with historical control. Toxicity profile was not different from other bevacizumab-containing regimens, including hypertension, GI perforation, GI bleeding, and thromboembolic events.

Table 7 Ongoing clinical trials investigating bevacizumab for biliary duct carcinoma and gallbladder cancer

\begin{tabular}{|c|c|c|c|c|}
\hline Phase & Condition & Intervention and schedule & $\begin{array}{l}\text { Projected } \\
\text { accural }\end{array}$ & Investigator \\
\hline II & $\begin{array}{l}\text { Cholangiocarcinoma, Gallbladder } \\
\text { cancer }\end{array}$ & $\begin{array}{l}\text { Erlotinib } 150 \mathrm{mg} \text { daily, bevacizumab } \\
10 \mathrm{mg} / \mathrm{kg} \mathrm{q} 14 \mathrm{~d}\end{array}$ & 126 & $\begin{array}{l}\text { Rigshospitalet, } \\
\text { Denmark }\end{array}$ \\
\hline II & $\begin{array}{l}\text { Extrahepatic bile duct cancer, } \\
\text { gallbladder cancer }\end{array}$ & $\begin{array}{l}\text { Erlotinib daily bevacizumab q } 14 d \\
\text { (dose not specified) }\end{array}$ & 55 & Mayo Clinic \\
\hline 1 & $\begin{array}{l}\text { Hepatocellular carcinoma, } \\
\text { cholangiocarcinoma }\end{array}$ & $\begin{array}{l}\text { Proton radiotherapy } 3 \text { Gy per fraction } \times 20 \text {, } \\
\text { bevacizumab } 10 \mathrm{mg} / \mathrm{kg} \text { q } 14 \mathrm{~d}\end{array}$ & 30 & MD Anderson \\
\hline$\|$ & $\begin{array}{l}\text { Billiary tract adenocarcinoma, } \\
\text { Gallbladder adenocarcinoma }\end{array}$ & $\begin{array}{l}\text { Gemcitabine qI4d oxaliplatin ql4d } \\
\text { bevacizumab qI4d (dose not specified) }\end{array}$ & 37 & $\mathrm{MGH}$ \\
\hline$\|$ & $\begin{array}{l}\text { Biliary tract cancer, Gallbladder } \\
\text { adenocarcinoma }\end{array}$ & $\begin{array}{l}\text { Capecitabine oxaliplatin bevacizumab } \\
\text { q } 14 \text { d Radiation therapy for total } \\
28 \text { treatments (dose not specified) }\end{array}$ & 26 & $\mathrm{MGH}$ \\
\hline
\end{tabular}

Notes: Adapted from www.clinicaltrials.gov.

Abbreviations: MGH, Massachusetts General Hospital; 5-FU/LV, fluouracil and leucovorin; FOLFOX, 5-FU/LV/oxaliplatin; mFOLFOX, modified FOLFOX; FOLFIRI, 5-FU/LV/ irinotecan; CapeOX, capecitabine/oxaliplatin; IFL, irinotecan, 5-FU, and LV; mIFL, bolus IFL. 
Further optimization of the use of bevacizumab in gastric and GE junction cancers is warranted.

Two abstracts presented at the 2009 ASCO annual meeting are worth discussing. ${ }^{79,80}$ Based on the data obtained in the Shah phase II study, Kelsen and colleagues investigated modified docetaxel, cisplatin, fluorouracil (mDCF), and bevacizumab in metastatic GE junction adenocarcinoma as first-line therapy. ${ }^{79}$ The primary objective was to improve 6-month PFS from $43 \%$ (historical DCF control) to $63 \%$ with the addition of bevacizumab. Thirty-nine of 44 eligible patients had measurable disease. Overall RR was 67\% (95\% CI $50 \%$ to $81 \%$ ), 6-month PFS was $79 \%$ (95\% CI $68 \%$ to 93\%), median PFS was 12 months (95\% CI 8.8 to 16), and median OS was 16.2 months (95\% CI 11.4 to infinitiy) after a median follow-up of 12.3 months. This bevacizumabcontaining regimen provided a durable survival benefit without significant toxicities.

A similar regimen with docetaxel and oxaliplatin in combination with bevacizumab was investigated by El-Raves and colleagues. ${ }^{80}$ The primary endpoint was TTP. A total of 23 patients with locally advanced or metastatic gastric/GE junction cancer were enrolled. PR was confirmed in 10 (59\%) patients. However, in contrast to the safety report of the other mDCF plus bevacizumab trial, 3 GI perforations was reported in this trial. This alerted the investigators not to use bevacizumab outside clinical trials until more safety data are obtained.

\section{Neuroendocrine tumors (NET)}

Neuroendocrine tumors, also named gastroenteropancreatic NET (GEP-NET), are cancers arising from endocrine (hormonal) system and the nervous system. There are essentially two distinct categories: functional or secretory, and nonfunctional tumors. Secretory NETs are usually treated with somatostatin analog-based therapy such as octreotide for symptomatic control. In metastatic stage, chemotherapy generally has low response rate with short duration.

Chemotherapeutic agents tested in locoregionally advanced or metastatic NETs include doxorubicin, capecitabine, oxaliplatin, streptozocin, 5-FU, temozolomide, and dacarbazine as single agents or in combination. Early data suggest that single-agent bevacizumab for advanced carcinoid tumors inhibits tumor blood flow and increases PFS. ${ }^{81}$ At the 2008 ASCO annual meeting, Kunz and colleagues presented the interim analysis of a phase II trial investigating a combination of capecitabine, oxaliplatin, and bevacizumab for metastatic/unresectable NETs. ${ }^{82}$ This is a relatively well tolerated regimen for CRC. The primary endpoints were to determine median TTP and assess the toxicities associated with this regimen. Among 13 patients of a planned 37, 4 achieved a PR with durations of 33, 27+, $27+, 27+$ weeks, respectively. Toxicities were expected to be related to chemotherapeutic agents.

The Dana-Farber cancer institute also conducted a phase II trial to investigate the efficacy of bevacizumab in combination with temozolomide in advanced NET. ${ }^{83}$ This was a nonrandomized, open label, single-arm study. The primary endpoint was to assess the response to bevacizumab and temozolomide in metastatic NET. Secondary endpoints were assess TTP, PFS, and safety of this combination. The schedule of temozolomide was once daily for 1 week followed by 1 week off. Bevacizumab was given intravenously every 2 weeks. Restaging CT scan was performed every 8 weeks to assess response. Among 29 evaluable patients for efficacy, 4 achieved PR, which made the response rate of $24 \%$. Bevacizumab-related toxicities were not severe except one patient developed hypertension.

The question of whether octreotide has an anti-tumor effect in addition to symptomatic control was answered at the 2009 GI ASCO meeting. ${ }^{84}$ PROMID (Placebo-controlled prospective Randomized study on the antiproliferative efficacy of Octreotide LAR in patients with metastatic neuroendocrine MIDgut tumors) was the first randomized, double-blind, placebo-controlled, multicenter, phase IIIb study of long-acting release (LAR) octreotide in patients with metastatic NETs. ${ }^{84}$ Eighty-five eligible patients randomly received either octreotide LAR or placebo. Octreotide LAR significantly lengthened median TTP from 6 months in the placebo groups to 14.3 months (HR 0.34 ; 95\% CI 0.20 to $0.59 ; P=0.000072)$. Therefore, a trial to test bevacizumab and octreotide combination in metastatic NETs would be the next step.

\section{Future directions}

Bevacizumab has provided the proof of concept that angiogenesis is an important target for cancer therapy and is the first anti-VEGF monoclonal antibody to receive FDA approval. Bevacizumab has shown clinical efficacy in a variety of advanced malignancies including CRC, nonsmall cell lung cancer, breast cancer, kidney cancer, ovarian epithelial cancer, and hepatocellular carcinoma.

Although bevacizumab has an established role in the treatment of metastatic colon, breast, and lung cancer, but many questions remain about its use in other disease types and demographic groups. Future studies are designed to evaluate. Important questions in treatment of advanced cancers are the 
optimal dose and duration of treatment with bevacizumab. One of the main aims of the ongoing CAIRO3 trial is to investigate the possible benefit of maintenance therapy with bevacizumab and low-dose capecitabine after initial therapy with chemotherapy and bevacizumab in mCRC. Resolution of the question abut optimal dose, however, has become increasingly challenging, and it is the opinion of the authors that a determination of optimal duration is likely to have the greater effect on outcomes.

Biomarker studies are crucial in identifying patients who would most benefit from bevacizumab therapy. The ECOG 5202 trial is a unique ongoing phase III prospective study aimed at determining the role of chemotherapy in stage II colon cancer patients by assigning treatment base risk as determined by molecular profiling. Patients will be stratified to 'high risk' or 'low risk' based on microsatellite instability and loss of heterozygosity at $18 \mathrm{q}$. Patients deemed to be 'high risk' will be randomized to 5-FU/LV/oxaliplatin versus 5-FU/LV/oxaliplatin plus bevacizumab. Those patients deemed to be 'low risk' will be registered for observation only. Thus, identifying criteria for individualized treatment with bevacizumab may not only improve efficacy but, through better patient selection, could also limit the use of expensive therapies in populations not likely to benefit from them.

\section{Disclosures}

The authors declare no conflicts of interest.

\section{References}

1. Coultas L, Chawengsaksophak K, Rossant J. Endothelial cells and VEGF in vascular development. Nature. 2005;438:937-945.

2. Werther K, Christensen IJ, Brünner N, Nielsen HJ. Soluble vascular endothelial growth factor levels in patients with primary colorectal carcinoma. The Danish RANX05 Colorectal Cancer Study Group. Eur J Surg Oncol. 2000;26:657-662.

3. Seo Y, Baba H, Fukuda T, et al. High expression of vascular endothelial growth factor is associated with liver metastasis and a poor prognosis for patients with ductal pancreatic adenocarcinoma. Cancer. 2000;88:2239-2245

4. Ikeda N, Adachi M, Taki T, et al. Prognostic significance of angiogenesis in human pancreatic cancer. Br J Cancer. 1999;79:1553-1563.

5. Claudio PP, Russo G, Kumar CA, et al. pRb2/p130, vascular endothelial growth factor, p27(KIP1), and proliferating cell nuclear antigen expression in hepatocellular carcinoma: their clinical significance. Clin Cancer Res. 2004;10:3509-3517.

6. Folkman J. Tumor angiogenesis: therapeutic implications. $N$ Engl J Med. 1971;285:1182-1186.

7. Folkman J, Klagsbrun M. Angiogenic factors. Science. 1987;235: 442-447.

8. Folkman J. What is the evidence that tumors are angiogenesis dependent? J Natl Cancer Inst. 1990;82:4-6.

9. Augustin HG. Translating angiogenesis research into the clinic: the challenges ahead. Br J Radiol. 2003;76:(1):S3-S10.

10. Rhee J, Hoff PM. Angiogenesis inhibitors in the treatment of cancer. Expert Opin Pharmacother. 2005;6:1701-1711.
11. Ferrara N, Alitalo K. Clinical applications of angiogenic growth factors and their inhibitors. Nat Med. 1999;5:1359-1364.

12. Ignoffo RJ. Overview of bevacizumab: a new cancer therapeutic strategy targeting vascular endothelial growth factor. Am J Health Syst Pharm. 2004;61(21 Suppl 5):S21-S26.

13. Chase JL. Clinical use of anti-vascular endothelial growth factor monoclonal antibodies in metastatic colorectal cancer. Pharmacotherapy. 2008;28(11 Pt 2):23S-30S.

14. Kim KJ, Li B, Houck K, et al. The vascular endothelial growth factor proteins: identification of biologically relevant regions by neutralizing monoclonal antibodies. Growth Factors. 1992;7:53-64.

15. http://www.fda.gov/bbs/topics/NEWS/2004/NEW01027.html.

16. http://www.fda.gov/bbs/topics/NEWS/2006/NEW01488.html.

17. http://www.fda.gov/cder/Offices/OODP/whatsnew/ bevacizumab200802.html.

18. de Gramont A, Van Cutsem E. Investigating the potential of bevacizumab in other indications: metastatic renal cell, non-small cell lung, pancreatic and breast cancer. Oncology. 2005;69 Suppl 3:46-56.

19. Burris H 3rd, Rocha-Lima C. New therapeutic directions for advanced pancreatic cancer: targeting the epidermal growth factor and vascular endothelial growth factor pathways. Oncologist. 2008;13:289-298.

20. Jemal A, Siegel R, Ward E, et al. Cancer statistics. CA Cancer J Clin. 2008;58:71-96.

21. Goldberg RM, Sargent DJ, Morton RF, et al. A randomized controlled trial of fluorouracil plus leucovorin, irinotecan, and oxaliplatin combinations in patients with previously untreated metastatic colorectal cancer. J Clin Oncol. 2004;22:23-30.

22. Cassidy J, Tabernero J, Twelves C, et al. XELOX (capecitabine plus oxaliplatin): active first-line therapy for patients with metastatic colorectal cancer. J Clin Oncol. 2004;22:2084-2091.

23. Saltz LB, Cox JV, Blanke C, et al. Irinotecan plus fluorouracil and leucovorin for metastatic colorectal cancer. $N$ Engl J Med. 2000; 343:905-914.

24. Tournigand C, Andre T, Achille E, et al. FOLFIRI followed by FOLFOX or the recer sequence in advanced colorectal cancer: a randomized GERCOR study. J Clin Oncol. 2004;22:229-237.

25. Colucci G, Gebbia V, Paoletti G, et al. Phase III randomized trial of FOLFIRI versus FOLFOX4 in the treatment of advanced colorectal cancer: a multicenter study of the Grouppo Oncologico Dell'Italia Meridionale. J Clin Oncol. 2005;23:4866-4875.

26. Kabbinavar F, Hurwitz HI, Fehrenbacher L, et al. Phase II, randomized trial comparing bevacizumab plus fluorouracil (FU)/leucovorin (LV) with $\mathrm{FU} / \mathrm{LV}$ alone in patients with metastatic colorectal cancer. $J$ Clin Oncol. 2003;21:60-65.

27. Hurwitz H, Fehrenbacher L, Hainsworth JD, et al. Bevacizumab in combination with fluorouracil and leucovorin: an active regimen for first-line metastatic colorectal cancer. J Clin Oncol. 2005;23: 3502-3508.

28. Kabbinavar FF, Schulz J, McCleod M, et al. Addition of bevacizumab to bolus fluorouracil and leucovorin in first line metastatic colorectal cancer: results of a randomized phase II trial. J Clin Oncol. 2005; 23:3697-3705.

29. Kabbinavar FF, Hambleton J, Mass RD, et al. Combination analysis of efficacy: the addition of bevacizumab to fluorouracil/leucovorin improves survival for patients with metastatic colorectal cancer. J Clin Oncol. 2005;23:3705-3712.

30. Hurwitz H, Fehrenbacher L, Novotny W, et al. Bevacizumab plus irinotecan, fluorouracil, and leucovorin for metastatic colorectal cancer. N Engl J Med. 2004;350(23):2335-2342.

31. Fuchs CS, Marshall J, Mitchell E, et al. Randomized, controlled trial of irinotecan plus infusional, bolus, or oral fluoropyrimidines in firstline treatment of metastatic colorectal cancer: results from the BICC-C Study. J Clin Oncol. 2007;25:4779-4786.

32. Fuchs CS, Marshall J, Barrueco J, et al. Randomized, controlled trial of irinotecan plus infusional, bolus, or oral fluoropyrimidines in firstline treatment of metastatic colorectal cancer: updated results from the BICC-C study. J Clin Oncol. 2008;26:689-690. 
33. Saltz LB, Clarke S, Díaz-Rubio E, et al. Bevacizumab in combination with oxaliplatin-based chemotherapy as first-line therapy in metastatic colorectal cancer: a randomized phase III study. J Clin Oncol. 2008;26:2013-2019.

34. Hochster H, Hart LL, Ramanathan RK, et al. Safety and efficacy of oxaliplatin/fluoropyrimidine regimens with or without bevacizumab as first-line treatment of metastatic colorectal cancer (mCRC): final analysis of the TREE study. J Clin Oncol. 2008;26:3523-3529. Erratum in: J Clin Oncol. 2008;26:4697.

35. Hochster HS, Grothey A, Shpilsky A, et al. Effect of intravenous (IV) calcium and magnesium $(\mathrm{Ca} / \mathrm{Mg})$ versus placebo on response to FOLFOX+bevacizumab (BEV) in the CONcePT trial; 2008 Gastrointestinal Cancers Symposium. Abstract 280.

36. Giantonio BJ, Catalano PJ, Meropol NJ, et al. Bevacizumab in combination with oxaliplatin, fluorouracil, and leucovorin (FOLFOX4) for previously treated metastatic colorectal cancer: results from the Eastern Cooperative Oncology Group Study E3200. J Clin Oncol. 2007; 25:1539-1544.

37. Cunningham D, Humblet Y, Siena S, et al. Cetuximab monotherapy and cetuximab plus irinotecan in irinotecan-refractory metastatic colorectal cancer. $N$ Engl J Med. 2004;351:337-345.

38. Saltz LB, Lenz HJ, Kindler HL, et al. Randomized phase II trial of cetuximab, bevacizumab, and irinotecan compared with cetuximab and bevacizumab alone in irinotecan-refractory colorectal cancer: the BOND-2 study. J Clin Oncol. 2007;25:4557-4561.

39. Hecht JR, Mitchell E, Chidiac T, et al. An updated analysis of the safety and efficacy of oxaliplatin (Ox)/bevacizumab (bev) \pm panitumumab (pmab) for firstline treatment (tx) of metastatic colorectal cancer (mCRC) from a randomized, controlled trial (PACCE). [abstract] In: Program and abstracts of the 2008 Gastrointestinal Cancers Symposium, Orlando, FL, January 25-27, 2008.

40. Hecht JR, Mitchell E, Chidiac T, et al. Interim results from PACCE: Irinotecan (iri)/bevacizumab (bev) \pm panitumumab as first-line treatment for metastatic colorectal cancer. [abstract] In: Proceedings and abstracts of the 2008 Gastrointestinal Cancers Symposium, Orlando, FL, January 25-27, 2008.

41. Tol J, Koopman M, Rodenburg CJ, et al. A randomised phase III study on capecitabine, oxaliplatin and bevacizumab with or without cetuximab in first-line advanced colorectal cancer, the CAIRO2 study of the Dutch Colorectal Cancer Group (DCCG). An interim analysis of toxicity. Ann Oncol. 2008;19:734-738.

42. Tol J, Koopman M, Cats A, et al. Chemotherapy, bevacizumab, and cetuximab in metastatic colorectal cancer. $N$ Engl J Med. 2009;360: 563-572.

43. Combination chemotherapy and bevacizumab with or without erlotinib in treating patients with metastatic colorectal cancer that cannot be removed by surgery. www.clinicaltrials.gov. ClinicalTrials.gov identifier: NCT00265824.

44. Irinotecan and Cetuximab with or without bevacizumab in treating patients with metastatic colorectal cancer that progressed during first-line therapy. http://clinicaltrials.gov/ct2/show/ NCT00499369.

45. Meyerhardt JA, Stuart K, Fuchs CS, et al. Phase II study of FOLFOX, bevacizumab and erlotinib as first-line therapy for patients with metastatic colorectal cancer. Ann Oncol. 2007;18:1185-1189.

46. Allegra CJ, Yothers G, O'Connell MJ, et al. Initial safety report of NSABP C-08, a randomized phase III study of modified 5-fluorouracil (5-FU)/leucovorin (LCV) and oxaliplatin (OX) (mFOLFOX6) with or without bevacizumab (bev) in the adjuvant treatment of patients with stage II/III colon cancer. 2008 ASCO annual meeting. J Clin Oncol. 2008;26(May 20 Suppl; abstr 4006).

47. Wolmark N, Yothers G, O'Connell MJ, et al. A phase III trial comparing mFOLFOX6 to mFOLFOX6 plus bevacizumab in stage II or III carcinoma of the colon: Results of NSABP Protocol C-08. JClin Oncol. 2009;27:18s(suppl; abstr LBA4).

48. Jemal A, Siegel R, Ward E, et al. Cancer statistics, 2009. CA Cancer J Clin. 2009;59:225-249.
49. Burris HA 3rd, Moore MJ, Andersen J, et al. Improvements in survival and clinical benefit with gemcitabine as first-line therapy for patients with advanced pancreas cancer: a randomized trial. J Clin Oncol. 1997;15:2403-2413.

50. Moore MJ, Goldstein D, Hamm J, et al. Erlotinib plus gemcitabine compared with gemcitabine alone in patients with advanced pancreatic cancer: a phase III trial of the National Cancer Institute of Canada Clinical Trials Group. J Clin Oncol. 2007;25:1960-1966.

51. Kindler HL, Friberg G, Singh DA, et al. Phase II trial of bevacizumab plus gemcitabine in patients with advanced pancreatic cancer. $J$ Clin Oncol. 2005;23:8033-8040.

52. Kim GP, Oberg A, Kabat B, et al. NCCTG phase II trial of bevacizumab, gemcitabine, oxaliplatin in patients with metastatic pancreatic adenocarcinoma. [abstract] $J$ Clin Oncol. 2006; ASCO Annual Meeting Proceedings Part I. Vol 24, No. 18S (June 20 Suppl):4113.

53. Kim GP, Oberg AL, Foster NR, et al. Phase II trial of bevacizumab, gemcitabine, oxaliplatin in patients with metastatic pancreatic adenocarcinoma. [abstract] J Clin Oncol. 2007; ASCO Annual Meeting Proceedings Part I. Vol 25, No. 18S (June 20 Suppl):4553.

54. Louvet C, Labianca R, Hammel P, et al. Gemcitabine in combination with oxaliplatin compared with gemcitabine alone in locally advanced or metastatic pancreatic cancer: results of a GERCOR and GISCAD phase III trial. J Clin Oncol. 2005;23:3509-3516.

55. Heinemann V, Quietzsch D, Gieseler F, et al. Randomized phase III trial of gemcitabine plus cisplatin compared with gemcitabine alone in advanced pancreatic cancer. J Clin Oncol. 2006;24:3946-3952.

56. Heinemann V, Labianca R, Hinke A, Louvet $\mathrm{C}$. Increased survival using platinum analog combined with gemcitabine as compared to singleagent gemcitabine in advanced pancreatic cancer: pooled analysis of two randomized trials, the GERCOR/GISCAD intergroup study and a German multicenter study. Ann Oncol. 2007;18:1652-1659.

57. Heinemann V, Boeck S, Hinke A, Labianca R, Louvet C. Meta-analysis of randomized trials: evaluation of benefit from gemcitabine-based combination chemotherapy applied in advanced pancreatic cancer. BMC Cancer. 2008;8:82.

58. Astsaturov NJ, Meropol NJ, Alpaugh RK, et al. A randomized phase II and coagulation study of bevacizumab alone or with docetaxel in patients with previously treated metastatic pancreatic adenocarcinoma. [abstract] J Clin Oncol. 2007; ASCO Annual Meeting Proceedings. Part I. Vol. 25, No. 18S (June 20 Suppl):4556.

59. Kindler HL, Gangadhar T, Karrison T, et al. Final analysis of a randomized phase II study of bevacizumab (B) and gemcitabine (G) plus cetuximab (C) or erlotinib (E) in patients (pts) with advanced pancreatic cancer (PC). [abstract] J Clin Oncol. 2008; ASCO Annual Meeting Proceedings:Abstract 4502.

60. Kindler HL, Niedzwiecki D, Hollis D, et al. A double-blind, placebocontrolled, randomized phase III trial of gemcitabine (G) plus bevacizumab (B) versus gemcitabine plus placebo (P) in patients (pts) with advanced pancreatic cancer (PC): A preliminary analysis of Cancer and Leukemia Group B (CALGB). J Clin Oncol. 2007;25:18s(Suppl): Abstract 4508.

61. Van Cutsem E, Vervenne WL, Bennouna J, et al. Phase III Trial of bevacizumab in combination with gemcitabine and erlotinib in patients with metastatic pancreatic cancer. J Clin Oncol. 2009;27:2231-2237.

62. Yamanaka Y, Friess H, Kobrin MS, et al. Coexpression of epidermal growth factor receptor and ligands in human pancreatic cancer is associated with enhanced tumor aggressiveness. Anticancer Res. 1993;13:565-569.

63. Longo R, Cacciamani F, Naso G, et al. Pancreatic cancer: From molecular signature to target therapy. Crit Rev Oncol Hematol. 2008;68:197-211

64. Llovet JM, Ricci S, Mazzaferro V, et al. Sorafenib in advanced hepatocellular carcinoma. N Engl J Med. 2008;359:378-390.

65. Finn RS, Bentley G, Britten CD, Amado R, Busuttil RW. Targeting vascular endothelial growth factor with the monoclonal antibody bevacizumab inhibits human hepatocellular carcinoma cells growing in an orthotopic mouse model. Liver Int. 2009;29:284-290. 
66. Siegel AB, Cohen EI, Ocean A, et al. Phase II trial evaluating the clinical and biologic effects of bevacizumab in unresectable hepatocellular carcinoma. J Clin Oncol. 2008;26:2992-2998.

67. Zhu AX, Blaszkowsky LS, Ryan DP, et al. Phase II study of gemcitabine and oxaliplatin in combination with bevacizumab in patients with advanced hepatocellular carcinoma. J Clin Oncol. 2006;24:1898-1903.

68. Zhu AX, Holalkere NS, Muzikansky A, Horgan K, Sahani DV. Early antiangiogenic activity of bevacizumab evaluated by computed tomography perfusion scan in patients with advanced hepatocellular carcinoma. Oncologist. 2008;13:120-125.

69. Thomas MB, Morris JS, Chadha R, et al. Phase II trial of the combination of bevacizumab and erlotinib in patients who have advanced hepatocellular carcinoma. J Clin Oncol. 2009;27:843-850.

70. Kaseb AO, Iwasaki M, Javle M, et al. Biological activity of bevacizumab and erlotinib in patients with advanced hepatocellular carcinoma (HCC). J Clin Oncol. 2009;27(15s Suppl):Abstract 4522.

71. Ong LC, Song IC, Jin Y, et al. Effective inhibition of xenografts of hepatocellular carcinoma (HepG2) by rapamycin and bevacizumab in an intrahepatic model. Mol Imaging Biol. 2009;11:334-342.

72. Antiangiogenic treatment of HCC with bevacizumab and Rad001. http://www.clinicaltrials.gov/ct2/show/NCT00775073.

73. Tai CJ, Chiou HY, Wu CH, Pan S, Liu JD. Rapid resolution of liver metastasis from cholangiocarcinoma after bevacizumab with cisplatin and high-dose fluorouracil plus leucovorin. Onkologie. 2006;29:179-180.

74. Holen KD, Mahoney MR, LoConte NK, et al. Efficacy report of a multicenter phase II trial testing a biologic-only combination of biweekly bevacizumab and daily erlotinib in patients with unresectable biliary cancer (BC): A Phase II Consortium (P2C) study. J Clin Oncol. 2008;26(Meeting Abstracts):Abstract 4522.

75. Eroglu A, Demirci S, Ayyildiz A, et al. Serum concentrations of vascular endothelial growth factor and nitrite as an estimate of in vivo nitric oxide in patients with gastric cancer. Br J Cancer. 1999;80:1630-1634.

76. Karayiannakis A, Syrigos KN, Polychronidis A, et al. Circulating VEGF levels in the serum of gastric cancer patients. Ann Surg. 2002;236:37-42.
77. Ninomiya S, Inomata M, Tajima M, et al. Effect of Bevacizumab, a Humanized Monoclonal Antibody to Vascular Endothelial Growth Factor, on Peritoneal Metastasis of MNK-45P Human Gastric Cancer in Mice. J Surg Res. 2009;154:196-202.

78. Shah MA, Ramanathan RK, Ilson DH, et al. Multicenter phase II study of irinotecan, cisplatin, and bevacizumab in patients with metastatic gastric or gastroesophageal junction adenocarcinoma. J Clin Oncol. 2006;24:5201-5206.

79. Kelsen D, Jhawer M, Ilson D, et al. Analysis of survival with modified docetaxel, cisplatin, fluorouracil (mDCF), and bevacizumab (BEV) in patients with metastatic gastroesophageal (GE) adenocarcinoma: Results of a phase II clinical trial. J Clin Oncol. 2009;27(15s Suppl): Abstract 4512 .

80. El-Rayes BF, Patel B, Zalupski M, et al. A phase II study of bevacizumab, docetaxel, and oxaliplatin in gastric and GEJ cancer. J Clin Oncol. 2009;27(15s Suppl):Abstract 4563.

81. Yao JC, $\mathrm{Ng} \mathrm{C}$, Hoff PM, et al. Improved progression free survival (PFS), and rapid, sustained decrease in tumor perfusion among patients with advanced carcinoid treated with bevacizumab. J Clin Oncol. 2005; ASCO Annual Meeting Proceedings. Vol 23, No. 16S, Part I of II (June 1 Suppl):Abstract 4007

82. Kunz PL, Kuo T, Kaiser HL, et al. A phase II study of capecitabine, oxaliplatin, and bevacizumab for metastatic or unresectable neuroendocrine tumors: Preliminary results. J Clin Oncol. 2008; 26(May 20 Suppl):Abstract 15502.

83. Kulke MH, Stuart K, Earle CC, et al. A phase II study of temozolomide and bevacizumab in patients with advanced neuroendocrine tumors. $J$ Clin Oncol. 2006; ASCO Annual Meeting Proceedings Part I. Vol 24, No. 18S (June 20 Suppl):Abstract 4044

84. Arnold R, Müller H, Schade-Brittinger C, et al. Placebo-controlled, double-blind, prospective, randomized study of the effect of octreotide LAR in the control of tumor growth in patients with metastatic neuroendocrine midgut tumors: A report from the PROMID study group. J Clin Oncol. 2009;27(15s Suppl):Abstract 4508.
Biologics: Targets \& Therapy

\section{Publish your work in this journal}

Biologics: Targets \& Therapy is an international, peer-reviewed journal focusing on the patho-physiological rationale for and clinical application of Biologic agents in the management of autoimmune diseases, cancers or other pathologies where a molecular target can be identified. This journal is indexed on PubMed Central, CAS,

\section{Dovepress}

EMBase, Scopus and the Elsevier Bibliographic databases. The manuscript management system is completely online and includes a very quick and fair peer-review system, which is all easy to use. Visit http://www.dovepress.com/testimonials.php to read real quotes from published authors. 